\title{
Spectrum Allocation in Cognitive Radio: A PSO-based Approach
}

\author{
Subhashree Mishra', Santwana Sagnika ${ }^{2 *}$, Sudhansu Sekhar Singh', \\ Bhabani Shankar Prasad Mishra²
}

\footnotetext{
1 School of Electronics Engineering, Kalinga Institute of Industrial Technology Deemed to be University, 751024-Bhubaneswar, India 2 School of Computer Engineering, Kalinga Institute of Industrial Technology Deemed to be University, 751024-Bhubaneswar, India

*Corresponding author, e-mail: santwana.sagnika@gmail.com
}

Received: 02 September 2018, Accepted: 16 November 2018, Published online: 10 January 2019

\begin{abstract}
Cognitive radio systems have taken a fore-running position in the wireless communication technology. With most of the communication taking place through multi-carrier systems, the allocation of available spectrum to various carriers is a prominent issue. Since cognitive systems provide an environment of dynamic spectrum allocation, it becomes necessary to perform dynamic spectrum allocation swiftly with due consideration of parameters, like power consumption, fair distribution and minimal error. This paper considers a Particle Swarm Optimization-based approach, popularly used for solving large problems involving complex solution spaces to reach an optimal solution within feasible time. The mentioned spectrum allocation problem has been solved using PSO with a view to maximize the total transfer rate of the system, within specified constraints of maximum error rate, maximum power consumption and minimum transfer rate per user. The results have been compared with the existing Genetic Algorithm-based approach and have proved to be more effective.
\end{abstract}

\section{Keywords}

cognitive radio, spectrum allocation, Particle Swarm Optimization, Genetic Algorithm, multi-carrier systems

\section{Introduction}

Wireless communication is on a rapid upward growth curve, attracting more and more investment. Since the number of users keeps on increasing, the available radio spectrum, being a limited resource, is under constant demand. So there is always a requirement of obtaining maximum utilization of available spectrum at any instance of time. Currently the spectrum is distributed as an exclusive fixed bandwidth to a licensed user. Hence it is utilized only when the user utilizes it [1]. This becomes a cause of a non-optimal use of the entire spectrum available, since some users maybe experiencing heavy traffic while others may be using very less. Besides, with change in technology, the required bandwidth for different applications keeps on changing. So, the concept of dynamic spectrum allocation has come to the fore, which refers to changing the allotted spectrum to different users as per the requirement during data transmission through the allocated channels. In other words, spectrum allocation is better performed by considering it as a time-variant system. This dynamic spectrum allocation mechanism is popularly known as cognitive radio $[2,3]$.
Cognitive radio is a software-based radio system which intelligently performs tuning of transmission factors, like operational frequency, waveforms, etc., in a dynamic situation, by sensing and learning from the environment [4]. Its main job is to identify the unused portion of the spectrum of any primary user and take a decision on temporarily allocating it to other secondary users, while making sure that the interference caused to the primary user is avoided and it is re-allocated to the primary user on a priority basis whenever the need arises. Hence, cognitive radio performs spectrum sensing, allocation, resource optimization and mobility [5]. Fig. 1 represents these activities in the form of a cognitive cycle.

Spectrum sensing refers to the monitoring of the external environment by the cognitive radio, keeping track of the usage behavior of different primary users and locating spectrum holes. Considering this information, the allocation of spectrum is done and the parameters set accordingly to provide the best quality of service and minimize interference. The optimal resource utilization is tried to 


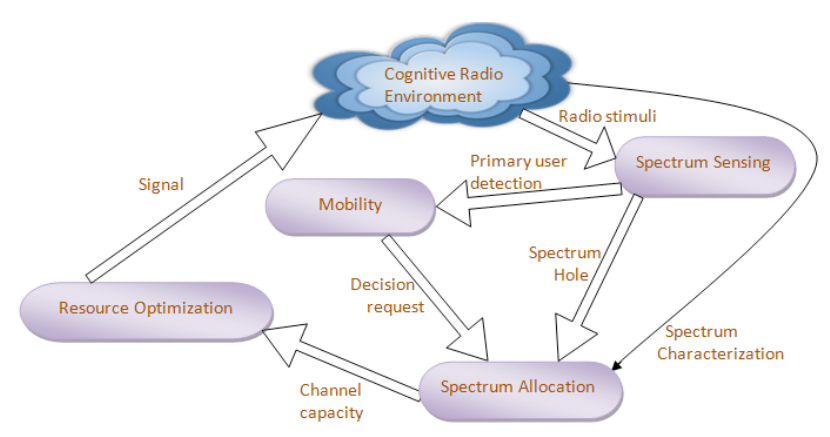

Fig. 1 The cognitive cycle

be achieved by balancing between the best efficiency of transmission and the sharing of spectrum among different users. Mobility also has to be maintained, i.e. whenever the primary user needs its allocated spectrum, it has to be made available by de-allocating from any secondary user who might be using it, so that disruption caused to the primary user is negligible. This process should be completed in real-time; hence it is the task of the cognitive radio system to continually observe the environment for the best possible free spectrum area $[6,7]$.

Recently, swarm-based and evolutionary techniques have been gaining much reputation in solving intractable problems, due to their ability to simultaneously search large spaces for obtaining optimal solutions, which otherwise are infeasible to be solved by traditional algorithms. Their ability to heuristically solve problems in real-time have made the preferred choice for scientific and business purposes.

Genetic Algorithm (GA) is a very simple and effective mechanism that has been applied to solving optimization problems. Similarly, the initially popular swarm-based techniques like Artificial Bee Colony (ABC), Particle Swarm Optimization (PSO), etc. and the more recent ones like Cat Swarm Optimization (CSO), Bat Algorithm (BA), etc. have been employed to solve such problems [8, 9].

In this paper, the authors have implemented a solution for the spectrum allocation problem in cognitive radio using PSO. A proper mapping of resources to devices has been obtained keeping certain constraints in consideration. The performance is compared with the existing GA-based approach and found to give more optimal solutions in lesser number of iterations.

\section{Literature survey}

Proper and optimal allocation of spectrum has been a trending area of research in recent times. A lot of work has been to done to find ways to allocate spectrum in a quick as well as efficient manner. Evolutionary algorithms have also been employed to serve this purpose. This section reviews the different mechanisms and approaches that have been adopted till date in this field.

ArunKumar and Reshma [4] have implemented a solution to spectrum allocation using Markov model, Banker's deadlock avoidance algorithm in combination with the graph coloring problem. This method has ensured fairly distributed spectrum allocation quickly and efficiently.

Koroupi et al. [8] have approached the problem using the ACO technique. This method has worked well for dynamic networks, providing a fast and low-cost solution. The method has proved to be superior to the PSO approach as proposed in [9], but takes a slightly higher running time.

Zhao et al. [9] have designed spectrum allocation algorithms for cognitive radio using GA, Quantum GA and PSO. They have considered interference parameters applied on channel availabilities. These methods have displayed better performance than general graph coloring algorithms.

Liu et al. [10] developed the Maximal Independent Set algorithm to address this problem. This mechanism ensured a fair distribution of resources while requiring very less overhead on the network.

Pradhan [11] has applied GA, PSO and ABC in different modes of operation for allocating spectrum in a time-varying environment. Spectral interference is taken into consideration. The author finds better performance of ABC over PSO and GA.

Wang et al. [12] have developed a heuristic cost and connection based algorithm which performed spectrum allocation considering power control. The algorithm was found to show better performance than the existing Maximal Independent Set algorithm, both in terms of Quality of Services as well as the number of Satisfied Users, even on bad networks.

Liu et al. [13] proposed a technique using simulated annealing approach to determine the optimal spectrum assignment. This technique aims to optimize the cumulative utility under constraints of capacity, rate requirement and interference for various traffic classes. It performs better than the previous greedy migration method.

$\mathrm{Ni}$ et al. [14] used game theory for efficient and flexible spectrum assignment in cognitive radio networks. They have classified allocation problems into co-operative and non-cooperative models. Then they have identified the suitability of the various game theory models to the different kinds of spectrum allocation problems. This work 
serves as a guideline for selecting the best game theory model depending upon the problem in hand.

Hou et al. [15] have put forward a spectrum allocation method based on an improved GA. It aims to maximize the transfer rate of a system, under the constraints of error rate, power consumption and a fairness of spectrum distribution. It provides quite a noticeable improvement over the simple GA method.

Tang and Xin [16] have applied co-evolution chaotic PSO to maximize energy efficiency, under the constraints of interference power and total transmit power. Anandakumar and Umamaheswari [17] have performed efficient social cognitive handover using socially intelligent secondary users and integration of primary and secondary holes, by applying a SpecPSO technique.

$\mathrm{Xu}$ et al. [18] have targeted to maximize the average weighted sum rate of throughput, within the constraints of total power and probabilistic interference. They have applied a hybrid PSO (SVM+PSO) for effective optimization. In another work, Xu et al. [19] have minimized total power consumption for uplink resource allocation based on chance constrained programming, with the help of a hybrid QPSO (QPSO+RBFN) technique.

Based on the conclusions obtained in the existing literature, the authors have found that swarm-based algorithms are being extensively applied on optimization problems, and since optimal spectrum allocation is a major requirement in cognitive radio networks, swarm-based techniques are attractive enough to be applied to solve this problem. The authors' approach is described in the following sections.

\section{Problem description}

The spectrum allocation part of cognitive radio is basically about determining the best way in which alternative resources can efficiently and non-dominantly use the available regions of the entire spectrum on-demand, while making sure that the primary user can use its own spectrum without any intervention due to this sharing process. A fair distribution is also desirable in such a scenario. This problem has been re-casted to a NP-hard problem and suitable approaches have been designed to solve it, like game theory, graph coloring, etc. [8].

Cognitive radio networks consist of densely distributed base stations, multiple users and sensor nodes. A variety of factors affect the allocation of spectrum among secondary nodes, including bit error rate, available free carriers, transmission power, user needs, etc. In this paper, the authors consider the constraints of maximum bit error rate, maximum total power usage and minimum individual user transfer rate. The reason behind taking these constraints is because it ensures energy efficient mechanism with minimal error, along with a fair distribution of necessary spectrum among all users [11, 20, 21].

Thus, the aim of this paper is to find a best allocation of the available subcarriers to the different users. For any allocation, the transmission power of each subcarrier is calculated. Also, Multiple Quadrature Amplitude Modulation (MQAM) is used; hence the MQAM order is determined for the allocation. The total transfer rate of the system is therefore calculated. So the best allocation is the one for which the total transfer of the system has the maximum value. Besides, the above-mentioned constraints are needed to be taken into account while determining the allocation.

Consider a specific allocation represented as

$X=\left[\begin{array}{cccc}x_{11} & x_{12} & \cdots & x_{1 Q} \\ x_{21} & \vdots & \ddots & x_{2 Q} \\ \vdots & \vdots & \ddots & \vdots \\ x_{P 1} & x_{P 2} & \cdots & x_{P Q}\end{array}\right]$

where, $x_{p q}=\left(M_{p q}, T P_{p q}\right)$.

The total transfer rate of the system is given by

$T T R=B * \sum_{q=1}^{Q} \sum_{p=1}^{P} U_{p q}$

where $U_{p q}$ is given by

$U_{p q} \cong 0.5 * \log _{2} M_{p q}$.

Here, $B$ represents the subcarrier bandwidth. The system has $P$ users and $Q$ subcarriers. $U_{p q}$ is the utilization of spectrum and $M_{p q}$ is the QAM modulation order, $T P_{p q}$ is the transmission power, for the $p^{\text {th }}$ user and $q^{\text {th }}$ subcarrier.

Therefore, the aim of this work is

Maximize (TTR); subject to

$\left(B * \sum_{q=1}^{Q} U_{p q}\right) \geq T R_{\min }$

$E \leq E_{\max }$,

$T T P \leq T P_{\max }$.

Here, $T R_{\min }$ is the minimum transfer rate for any user in Kbits/s, $E_{\max }$ is the maximum permissible bit error rate, and $T P_{\max }$ is the maximum total power usage of the system. $E$ is the average bit error rate over the system, and TTP is the total transmission power of the system. 
$E=\operatorname{avg}\left(E_{p q}\right) ; \forall p, q$, such that $M_{p q} \neq 0$

$E_{p q}=\left(\frac{4}{\log _{2} M_{p q}}\right)\left(1-\frac{1}{\sqrt{\log _{2} M_{p q}}}\right) \operatorname{erfc}\left(\sqrt{\frac{3 * \log _{2} M_{p q} * T P_{p q}}{\left(M_{p q}-1\right) * Q}}\right)$.

Here, $\operatorname{erfc}$ is the complementary error function.

$T T P=\sum_{q=1}^{Q} \sum_{p=1}^{P} T P_{p q} ; \forall p, q$, such that $M_{p q} \neq 0$.

Equation (4) represents the fitness function for the technique described further in this paper.

\section{Proposed technique}

\subsection{Particle Swarm Optimization}

Particle Swarm Optimization (PSO) is a swarm-based technique focused on solving optimization problems. It was designed by Kennedy and Eberhart in 1995 [22]. It is based on swarm intelligence and draws on the behavior of birds, who habitually scatter to different locations when they start searching for food. Depending on their current location, they try to trace food sources. Birds closer to the source can detect the source and communicate this information to other birds so that they can move nearer to the source. Hence, after a number of such movements the birds will congregate to the food source. The cooperation among the birds is essential for finding the solution [23, 24].

The PSO method emulates this behavior of birds in a computing environment to seek optimal solutions over large spaces. It uses a population of particles, representing birds, which start at random positions and find their closeness to the solution by evaluating a well-defined fitness function. Each particle finds its local best position, and the global best position among all particles is determined. Based on these values, the velocities of the birds are decided, and the new positions are obtained using these velocities. The involvement of local best and global best positions ensures the coordination and exchange of information among particles and also makes sure that the particles are directed closer to the solution with each step. The particles eventually converge to the solution by repeating these steps till certain stopping criteria are reached $[25,26]$.

The following is a general algorithm of PSO.

1 Initialize the population with random positions and velocities

2 Assess fitness for all particles

$3 \quad$ Find personal best (pbest) for individual particles and global best (gbest) for all particles
$4 \quad$ While stopping criteria is not reached

$5 \quad$ Compute velocity for particles using Eq. (7)

6 Renew positions of particles using Eq. (8)

7 Assess fitness for all particles

8 If fitness value for a particle is superior to its pbest

9 Replace pbest with current fitness

10 End if

11 Find gbest among all particles

12 End while

Here, the position and velocity of the particles is determined as follows.

$$
\begin{aligned}
V_{i}^{\text {new }} & =w * V_{i}^{\text {old }}+a_{1} * r_{1}\left(\text { pbest }_{i}^{\text {old }}-P_{i}^{\text {old }}\right) \\
& +a_{2} * r_{2} *\left(\text { gbest }_{i}^{\text {old }}-P_{i}^{\text {old }}\right) \\
P_{i}^{\text {new }} & =P_{i}^{\text {old }}+V_{i}^{\text {new }} .
\end{aligned}
$$

In these equations, $V_{i}^{\text {old }}$ is the velocity of $i^{\text {th }}$ particle in the previous generation, $V_{i}^{\text {new }}$ is the velocity of $i^{\text {th }}$ particle in the current generation, $P_{i}^{\text {old }}$ is the position of $i^{\text {th }}$ particle in the previous generation, $P_{i}^{\text {new }}$ is the position of $i^{\text {th }}$ particle in the current generation, $a_{1}$ and $a_{2}$ are accelerating factors of local and global information respectively, $r_{1}$ and $r_{2}$ are random values between 0 and $1, w$ is the inertia weight.

\subsection{PSO for spectrum allocation}

In this paper, the authors propose an algorithm that uses PSO to address the spectrum allocation problem in cognitive radios as described in the Mathematical Model for spectrum allocation section. In the proposed mechanism, a population of particles is taken. Each particle represents a specific allocation of users to carriers as shown by the matrix X. The fitness of each particle is found out using Eq. (4). After finding pbest and gbest for each particle, the new velocities are calculated and the positions updated. This process is repeated till the best allocation is found, i.e. the allocation which gives the maximum total transfer rate, under the specified constraints of bit error rate, individual transfer rate and total power usage.

The flowchart in Fig. 2 represents the steps of the proposed technique.

\section{Implementation}

\subsection{Experimental setup}

The proposed method has been executed using MATLAB on a laptop having Intel i5 core processor and a 4 GB RAM. For PSO technique, the population size is taken as 


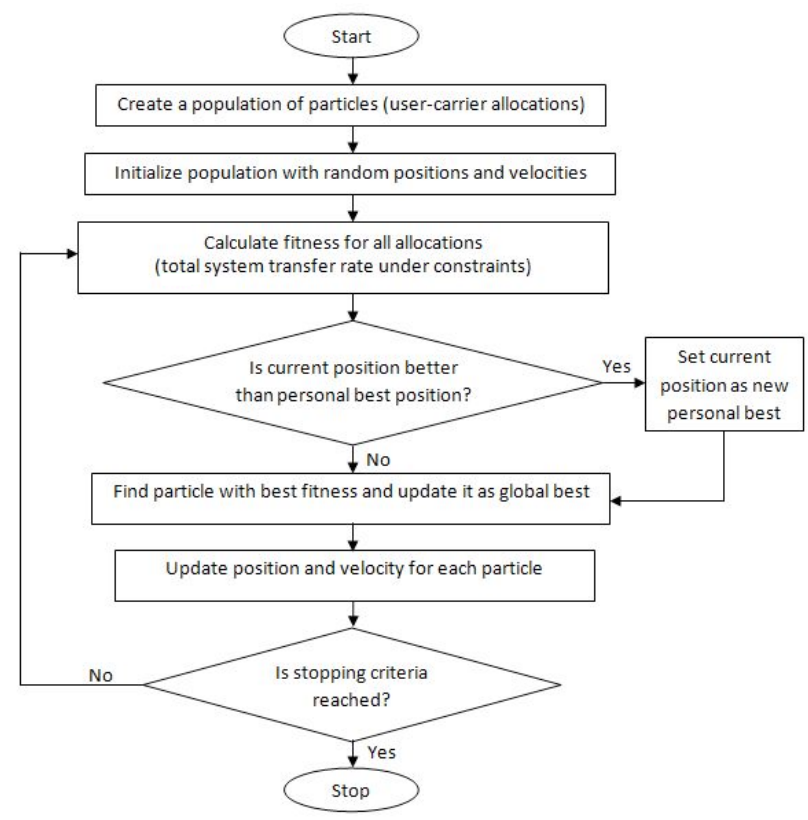

Fig. 2 Steps of the proposed algorithm

50 and the number of iterations is varied from 0 to 200 in steps of 20. The number of users is assumed to be 50 and the available carriers is taken as 2000 . The bandwidth $B$ is set as $30 \mathrm{KHz}$. The QAM modulation order $M p q \in\{0$, $16,32,64,128,256\}$, that depends on not used, 16QAM, 32QAM, and so on. The order of the modulation used, and the transmission power $T P p q$, are generated randomly. $T R_{\text {min }}$, the minimum transfer rate is set to be as 64 Kbits/s. The maximum allowed bit error rate $E_{\max }$ is set as $0.0005 \%$. The maximum total power usage $T P_{\max }$ of the system is taken as $80 \mathrm{~W}$.

The PSO parameters have been tested with various values till the best results are obtained. The optimal settings include $a_{1}$ and $a_{2}$ both as 2 and $w$ as 0.6 , while $r_{1}$ and $r_{2}$ are randomly generated, as already mentioned.

\subsection{Results and analysis}

The simulation results are plotted in Figs. 3-5. The results show that a higher transfer rate is obtained with a faster execution time by following the proposed approach using PSO, as compared to the existing mechanisms, i.e. Improved GA (I-GA) and GA, as shown by Hou et al. in [15]. The proposed approach converges at approximately 60 iterations, which makes it a fast and efficient approach as well. This is due to the fact that the particles share the information among them, thereby rendering every consecutive generation a more optimal one than the previous. Also, by varying the number of users, it is seen that the proposed mechanism gives higher transfer rates than existing methods for any
Total transfer rate vs. No. of iterations

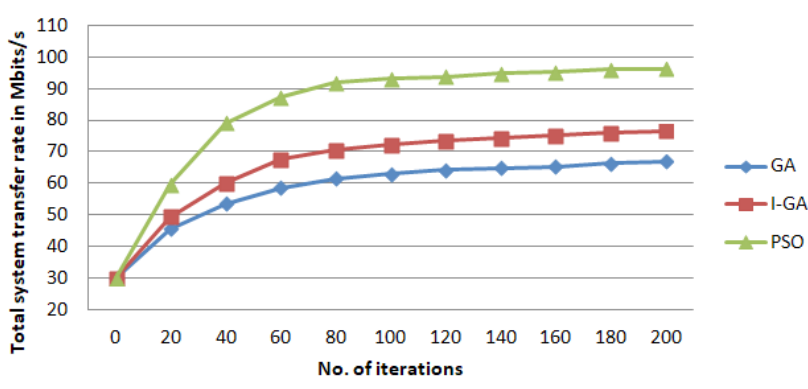

Fig. 3 Total system transfer rate vs. No. of iterations for GA, Improved GA and PSO, with no. of users $=50$

Total transfer rate vs. No. of Users

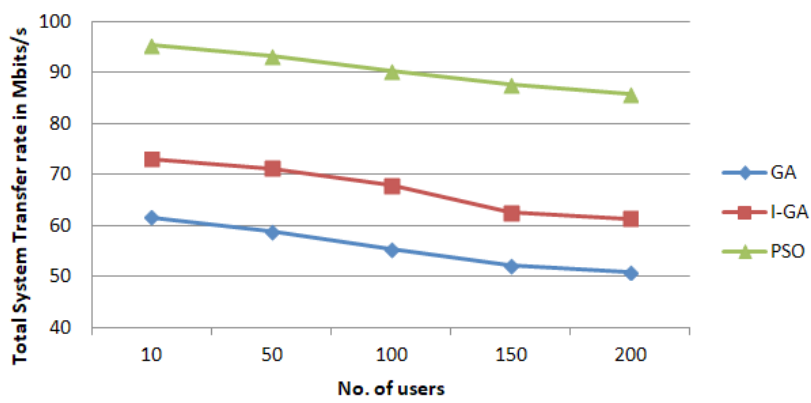

Fig. 4 Total System Transfer Rate vs. No. of users for GA, I-GA and PSO. PSO gives results at around 60 iterations.

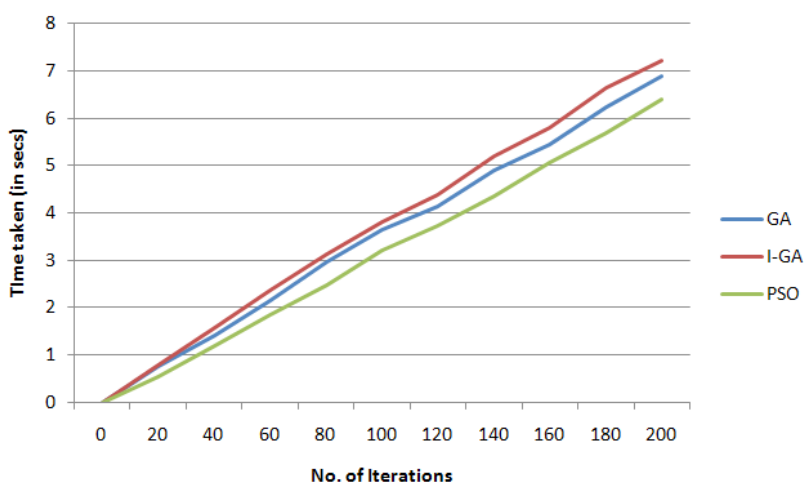

Fig. 5 Execution Time vs. No. of iterations for GA, I-GA and PSO.

given number of users. Hence, it is observed that the proposed approach using PSO is an effective and fast approach to perform spectrum allocation in cognitive radio networks.

The comparison is performed with GA and I-GA because these techniques are specifically implemented for maximizing the total system transfer rate. PSO techniques have been applied on different aspects of spectrum allocation. Tang and Xin [16] deal with maximizing energy efficiency using a co-evolution chaotic PSO, Anandakumar and Umamaheswari [17] focus on a social approach to cognitive handover using SpecPSO, Xu et al. [18] talk 
about maximizing average weighted sum rate of throughput, with the help of a hybrid PSO model. Xu et al. [19] minimize total power consumption using a RBFN based QPSO. Since these methods deal with objectives dissimilar to the problem in this paper, they are not considered for comparison. These methods can be applied to this paper's objective as a future work.

\section{Conclusions and future work}

In this paper, the authors have designed a spectrum allocation methodology for cognitive radio networks. The allocation is performed under constraints of bit error rate, total power usage and individual transfer rate. The method is

\section{References}

[1] Hazim, H. T., Shirsat, S. A. "Power Efficient Bandwidth Allocation for Cognitive LTE Networks", International Journal of Advanced Research in Computer Science and Software Engineering, 5(4), pp. 1068-1076, 2015.

[2] Sharma, A., Katoch, M. "Analysis of Various Spectrum Sensing Techniques in Cognitive Radio", International Journal of Advanced Research in Computer Science and Software Engineering, 5(5), pp. 1100-1104, 2015.

[3] Tragos, E. Z., Zeadally, S., Fragkiadakis, A. G., Siris, V. A. "Spectrum Assignment in Cognitive Radio Networks: A Comprehensive Survey", IEEE Communications Surveys \& Tutorials, 15(3), pp. 1108-1135, 2013.

https://doi.org/10.1109/SURV.2012.121112.00047

[4] Arun Kumar, B. R., Reshma, C. R. "Spectrum Scheduling using Markov Chain Model in Cognitive Radio Networks", IOSR Journal of Computer Engineering (IOSR-JCE), 17(4), pp. 112-115, 2015. https://doi.org/10.9790/0661-1742112115

[5] Lakhekar, M. V., Kotgire, S. L. "Interval Type-2 Fuzzy Logic System for Dynamic Spectrum Access in Cognitive Radio", International Journal of Computer Applications, 131(8), pp. 34-40, 2015. https://doi.org/10.5120/ijca2015907439

[6] Tabaković, Z. "A Survey of Cognitive Radio Systems", Croatian Post and Electronic Communications Agency, Jurišićeva 13, Zagreb, Croatia, 03, 2000.

[7] Akyildiz, I. F., Lee, W.-Y., Chowdhury, K. R. "CRAHNs: Cognitive Radio Ad Hoc Networks", Ad Hoc Networks, 7(5), pp. 810-836, 2009.

https://doi.org/10.1016/j.adhoc.2009.01.001

[8] Koroupi, F., Talebi, S., Salehinejad, H. "Cognitive radio networks spectrum allocation: An ACS perspective", Scientia Iranica D, 19(3), pp. 767-773, 2012.

https://doi.org/10.1016/j.scient.2011.04.029

[9] Zhao, Z., Peng, Z., Zheng, S., Shang, J. "Cognitive Radio Spectrum Allocation using Evolutionary Algorithms", IEEE Transactions on Wireless Communications, 8(9), pp. 4421-4425, 2009.

https://doi.org/10.1109/TWC.2009.080939 compared to existing methods, and it is found that it provides a higher transfer rate in real-time, and takes fewer number of iterations to find the optimal solution. This is justified due to information exchange mechanism implemented by PSO which makes it move faster towards reaching the solution. The simplicity and efficiency of the algorithm makes it an attractive alternative solution for performing spectrum allocation. The future scope of this work can include the integration of more constraints and multiple objectives, like interference, delay, price, energy, risk factors, etc. The applied PSO mechanism can also be improved or modified, and newer swarm-based techniques may be applied to make the process even faster and more efficient.

[10] Liu, Y., Mengxiong, J., Tan, X., Lu, F. "Maximal Independent Set Based Channel Allocation Algorithm in Cognitive Radios", In: IEEE Youth Conference on Information, Computing and Telecommunication, 2009,Beijing, China, pp. 78-81. https://doi.org/10.1109/YCICT.2009.5382422

[11] Pradhan, P. M. "Design of Cognitive Radio Engine using Artificial Bee Colony Algorithm", In: IEEE International Conference on Energy, Automation, and Signal (ICEAS), Beijing, China, 2011, pp. $1-4$. https://doi.org/10.1109/ICEAS.2011.6147139

[12] Wang, Y., Zhang, Z., Li, F., Chen, J. "A Novel Spectrum Allocation Algorithm for Cognitive Radio Networks", Procedia Engineering, 29, pp. 2776-2780, 2012.

https://doi.org/10.1016/j.proeng.2012.01.389

[13] Liu, Z. M., Nasser, N., Hassanein, H. S. "Intelligent spectrum assignment and migration in cognitive radio network", EURASIP Journal on Wireless Communications and Networking, 2013(200), pp. 1-13, 2013. https://doi.org/10.1186/1687-1499-2013-200

[14] Ni, Q., Zhu, R., Wu, Z., Sun, Y., Zhou, L., Zhou, B. "Spectrum Allocation Based on Game Theory in Cognitive Radio Networks", Journal of Networks, 8(3), pp. 712-722, 2013. https://doi.org/10.4304/jnw.8.3.712-722

[15] Hou, B., Zu, Y., Li, W., Liu, G., Ding, J. "Cognitive Radio Spectrum Allocation Strategy Based on Improved Genetic Algorithm", Communications and Network, 5, pp. 22-26, 2013. https://doi.org/10.4236/cn.2013.53B2005

[16] Tang, M., Xin, Y. "Energy efficient power allocation in cognitive radio network using coevolution chaotic particle swarm optimization", Computer Networks, 100, pp. 1-11, 2016. https://doi.org/10.1016/j.comnet.2016.02.010

[17] Anandakumar, H., Umamaheswari, K. "A bio-inspired swarm intelligence technique for social aware cognitive radio handovers", Computers and Electrical Engineering, 71, pp. 925-937, 2018. https://doi.org/10.1016/j.compeleceng.2017.09.016 
[18] Xu, L., Wang, J., Li, Y.-p, Li, Q., Zhang, X. "Resource allocation algorithm based on hybrid particle swarm optimization for multiuser cognitive OFDM network", Expert Systems with Applications, 42(20), pp. 7186-7194, 2015. https://doi.org/10.1016/j.eswa.2015.05.012

[19] Xu, L., Qian, F., Li, Y., Li, Q., Yang, Y.-w., Xu, J. "Resource allocation based on quantum particle swarm optimization and RBF neural network for overlay cognitive OFDM System", Neurocomputing, 173(3), pp. 1250-1256, 2016. https://doi.org/10.1016/j.neucom.2015.08.083

[20] Newman, T. R., Barker, B. A., Wyglinski, A. M., Agah, A., Evans, J. B., Minden, G. J. "Cognitive Engine Implementation for Wireless Multicarrier Transceivers", Wireless Communications \& Mobile Computing, Special Issue: Cognitive Radio, Software Defined Radio And Adaptive Wireless Systems, 7(9), pp. 1129-1142, 2007. https://doi.org/10.1002/wcm.486

[21] Chevillard, S. "The functions erf and erfc computed with arbitrary precision and explicit error bounds", Information and Computation, 216, pp. 72-95, 2012.

https://doi.org/10.1016/j.ic.2011.09.001
[22] Kennedy, J., Eberhart, R. "Particle Swarm Optimization", In: Proceedings of the ICNN'95 International Conference on Neural Networks, Perth, Australia, 1995, pp. 1942-1945. https://doi.org/10.1109/ICNN.1995.488968

[23] Bai, Q. "Analysis of Particle Swarm Optimization Algorithm", Computer and Information Science, 3(1), pp. 180-184, 2010.

[24] Katiyar, S. "A Comparative Study of Genetic Algorithm and the Particle Swarm Optimization", AKGEC International Journal of Technology, 2(2), pp. 21-24, 2010.

[25] Imran, M., Hashim, R., Khalid, N. E. A. "An Overview of Particle Swarm Optimization Variants", Procedia Engineering, 53, pp. 491-496, 2013. https://doi.org/10.1016/j.proeng.2013.02.063

[26] Sagnika, S., Bilgaiyan, S., Mishra, B. S. P. "Image Change Detection Using Particle Swarm Optimization", In: Jain, L., Patnaik, S., Ichalkaranje, N. (eds.) Intelligent Computing, Communication and Devices, Vol. 308, Springer, New Delhi, India, pp. 795-803, 2015. https://doi.org/10.1007/978-81-322-2012-1_86 